\title{
Topological Ring Currents and Bond Currents in Some Neutral and Anionic Altans and Iterated Altans of Corannulene and Coronene
}

\author{
Timothy K. Dickens*(i) and Roger B. Mallion \\ Peterhouse, Cambridge, CB2 1RD England, United Kingdom
}

ABSTRACT: The novel series of conjugated systems called altans, defined nearly a decade ago, was subsequently extended to multiple ("iterated") altans, and their magnetic properties were calculated by Monaco and Zanasi using the ab initio ipso-centric formalism. Such properties of the single ("mono") altans of corannulene and coronene, calculated by this sophisticated ab initio approach, had earlier been compared with those calculated via the rudimentary Hückel-London-Pople-McWeeny (HLPM) method-a parameter-free topological, quasi graph-theoretical approach requiring knowledge only of the conjugated system's molecular graph and the areas of its constituent rings. These investigations are here extended to double and triple altans. HLPM bond currents in several neutral mono altans are found to differ from those in the corresponding dianion only in those bonds that lie on the structures' perimeters, while the HLPM bond currents in all bonds in the neutral double and triple altans of corannulene and coronene are precisely the same as in the respective dianions. Some rationalization of these unexpected phenomena is offered in terms of the highest occupied molecular orbital (HOMO) and lowest unoccupied molecular orbital (LUMO) nature of the role played by the lone nonbonding orbital in each of the neutral species and its respective dianion.

\section{INTRODUCTION}

Monaco and Zanasi $^{1-4}$ (with Memoli ${ }^{3}$ ) have defined a novel series of conjugated systems that they call "altans". An altan $^{1-11}$ formally arises when an unsaturated hydrocarbon (called "the parent" or "the core") is regarded as being placed inside a $[4 n]$-annulene and then outgoing $\mathrm{C}-\mathrm{H}$ bonds from the core/parent are substituted by $\mathrm{C}-\mathrm{C}$ bonds that are connected to alternating carbon atoms of the outer annulenehence, the terminology "altan". ${ }^{1-11}$ The process is illustrated, for example, by the formation of altan-corannulene and altancoronene described in ref 5; other clear examples of the process of altanization are to be found in refs $8-10$, and the chemical aspects of the subject are reviewed in ref 11 . Synthetic work in this area has also very recently been forthcoming. ${ }^{12}$

The above-mentioned authors then used the ab initio ipsocentric formalism ${ }^{1-4,13}$ to calculate pictorial current-density maps for these structures and, in some cases, quantitative integrated current densities, which are the equivalent (in the ipso-centric approach) of individual bond-current intensities. The present authors have previously been concerned with how well these calculated magnetic properties, obtained via this sophisticated ab initio approach, are mimicked by the much more rudimentary Hückel ${ }^{14}-$ London $^{15}-$ Pople $^{16}-$ McWeeny $^{17}$ (HLPM) method, a topological, quasi graph-theoretical approach $^{18,19}$ that ostensibly relies on no parameters whatsoever and whose predictions depend only on a knowledge of the molecular graph ${ }^{20}$ of the conjugated system under study, as well as on the areas of its constituent rings. ${ }^{18,19}$ For reviews, please see refs $21-24$.

In a previous paper in this journal, ${ }^{5}$ we studied altancorannulene (structure $\mathbf{1}$ in Figure 1) and altan-coronene

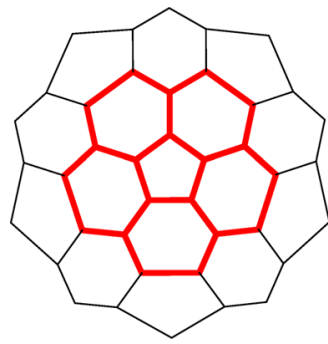

1

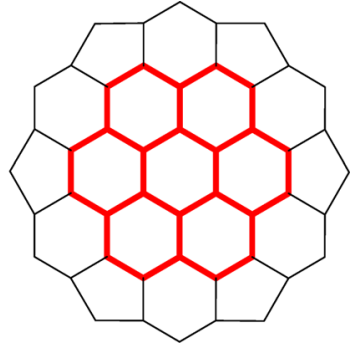

2
Figure 1. Molecular graphs ${ }^{20}$ of altan-corannulene (1) and altancoronene (2) showing their carbon-carbon connectivities. The carbon-carbon bonds of the parent conjugated systems (corannulene on the left, coronene on the right), which constitute the inner cores of these altan structures, are emphasized by being depicted in bold and in red.

(structure 2, in Figure 1). In this study, we examine the doubly altanized altan-altan-corannulene (structure 3 of Figure 2), which was introduced by Monaco and Zanasi in ref 4, as well as altan-altan-coronene (structure 4, in Figure 2)-these days

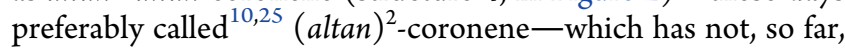
been considered. To compare our results with those of ref 4, we also examine the dianions of structures $1-4$. It is this comparison that unexpectedly yielded the major observations reported in this Article.

Received: July 17, 2018

Revised: August 18, 2018

Published: August 29, 2018 


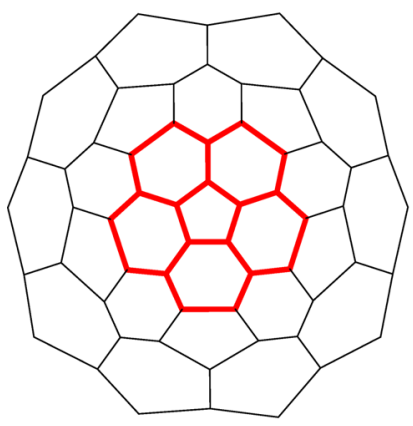

3

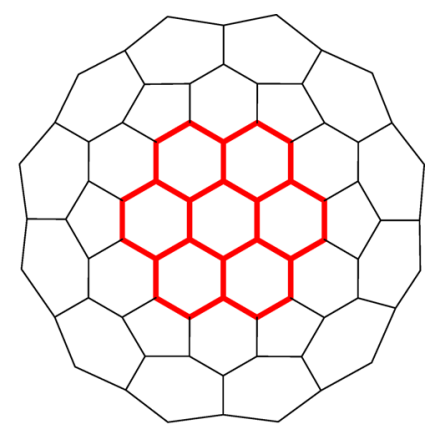

4
Figure 2. Molecular graphs ${ }^{20}$ of $(\text { altan })^{2}$-corannulene (3) and $(\text { altan })^{2}$-coronene (4) showing their carbon-carbon connectivities. The carbon-carbon bonds of the parent conjugated systems, which constitute the inner cores of these double altan structures, are emphasized by being displayed in bold and in red.

The mathematical properties of the altans have been studied by Monaco and Zanasi and, systematically, by Gutman. 8 Bašić and Pisanski have subsequently considered even higher altans (what have become known as iterated altans). ${ }^{10}$ Accordingly, in this Article, we further consider the topological ring-current and bond-current properties of the triple altan of corannulene (structure $\mathbf{5}$ in Figure 3) (more appropriately

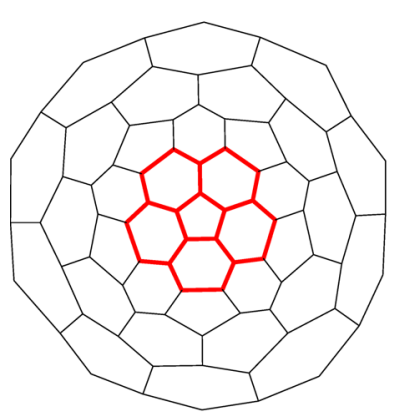

5

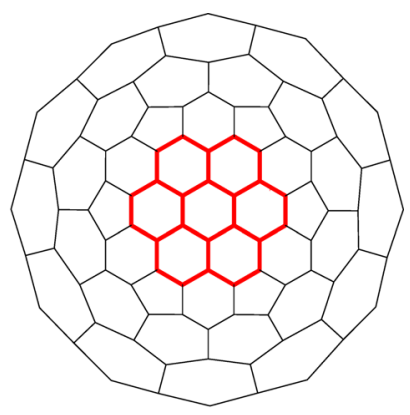

6
Figure 3. Molecular graphs ${ }^{20}$ of (left) the triple altan (5) of corannulene (best called ${ }^{10,25}$ (altan) ${ }^{3}$-corannulene) and (right) (altan $)^{3}$-coronene (6) showing their carbon-carbon connectivities. The carbon-carbon bonds of the parent conjugated systems that constitute the inner cores of these triple altan structures are again emphasized in bold and in red.

called $^{10,25}$ (altan $)^{3}$-corannulene) and the triple altan of coronene $\left((\text { altan })^{3}\right.$-coronene, structure 6 in Figure 3$)$, as well as their respective dianions. Some surprising results are obtained. First, though, we describe the method and the calculations.

\section{HLPM METHOD FOR CALCULATING RING CURRENTS AND BOND CURRENTS}

Overall Methodology. Ring-current intensities in structures 1-6 and their dianions were calculated using the standard HLPM ${ }^{14-17}$ topological $^{18,19,21-24}$ approach; eq 14 of ref 22 (explicitly documented as eq 4 in the next section of this Article) was applied, on the assumption of a planar geometry constituted from rings of carbon atoms whose areas are taken to be that of a regular polygon with the appropriate number of sides. This assumption may appear drastic, but previous studies $^{26,27}$ involving the HLPM approach-on conjugated systems that, like the altans, contain five-membered and sixmembered rings-have indicated that using ring areas obtained from X-ray crystallography, rather than idealized ones, changes calculated ring currents by at most $3 \% .^{26,27}$ Conceptually, numerically and computationally, this combination of conditions and assumptions that comprise what we call the "HLPM Topological" method ${ }^{18,19}$ is precisely equivalent ${ }^{21}$ to what is termed the "Current Density Hückel-London $(\mathrm{CD}-\mathrm{HL})$ " formulation in refs $28-30$. The symmetries ${ }^{31}$ assumed were $D_{5 h}$ (for 1, 3, and 5) ${ }^{32}$ and $D_{6 h}$ (for 2, 4, and $6)^{32}$ - please see refs 5 and 6 for a justification of this procedure when it is applied to nonplanar structures in which nonplanarity about any one bond is mild, even if the nonplanarity between well-separated parts of such structures may in practice be severe. ${ }^{33,34}$ Both ring currents and bond currents are here dimensionless, expressed, as they are, as ratios to the corresponding quantities calculated by the same method for benzene. The results are displayed in Figures 4-9 in the section headed Results and Preliminary Discussion.

Graph-Theoretical and Geometrical Quantities Arising in the HLPM Approach. For later analysis and discussion in this Article, it is necessary to consider certain graph-theoretical and geometrical quantities that arise in the HLPM formalism. ${ }^{21-24,35,36}$ We begin by recalling that method's graph-theoretical ${ }^{20} /$ Hückel molecular orbital $(\mathrm{HMO})^{14}$ basis. The adjacency matrix of the associated molecular graph ${ }^{20}$ (that is, the Hückel Hamiltonian matrix ${ }^{14}$ ) is first set up and diagonalized. This yields the set of eigenvectors, $\left\{\mathbf{c}_{J}\right\}_{J=1,2, \cdots, N}$ - the LCAO coefficients ${ }^{14}$-and the family of eigenvalues, $\left\{E_{J}\right\}_{J=1,2, \cdots, N}$ (which constitute the Hückel energy levels $\left.{ }^{14}\right)$. From these eigenvalues/energy levels, the orbital occupation numbers, $\left\{\nu_{J}\right\}_{J=1,2 \ldots, N}$, are devised by an application of the Aufbau principle. ${ }^{14}$

From these quantities, it is then straightforward to define the following:

(i) The Coulson bond order, $P_{r s}$, of the bond joining the carbon atoms labeled $r$ and $s$ in the associated molecular graph, as $^{14}$

$$
P_{(r s)}=\sum_{J=1}^{N} \nu_{J} \mathcal{c}_{J r} \mathcal{c}_{J s}
$$

(ii) The imaginary mutual bond-bond polarizability, $\bar{\pi}_{(r s)(t u)}$, between bonds $(r s)$ and $(t u)$ - the imaginary aspect arising as a result of the influence of the external magnetic field. This is the rate at which the imaginary part of the bond order, $P_{r s}$, of the bond $(r s)$ changes with respect to a variation in the imaginary part of the resonance integral, $\beta_{(t u)}$, of the bond $(t u)$. This imaginary mutual bond-bond polarizability is given by refs $17-19,21-24$, and 36 as

$$
\bar{\pi}_{(r s)(t u)}=\pi_{r s, t u}-\pi_{r s, u t}+\pi_{s r, u t}-\pi_{s r, t u}
$$

where

$$
\pi_{r s, t u}=\frac{2}{\beta} \sum_{I=1}^{M} \sum_{J=M+1}^{N} \frac{c_{I r} c_{J s} \mathcal{c}_{J t} c_{I u}}{E_{I}-E_{J}}
$$

Here, orbitals 1 to $M$ are assumed to be doubly occupied and orbitals $M+1$ to $N$ are entirely unoccupied.

These bond orders and polarizabilities are the graphtheoretical quantities that are needed in the calculation. Once these are known, the ring currents themselves are computed from an expression ${ }^{17-19,21-24,36}$ that brings together 


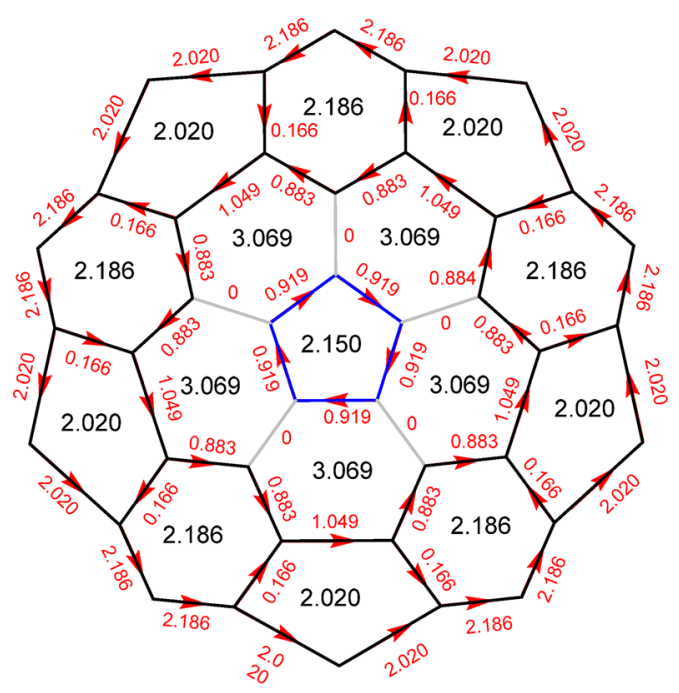

1

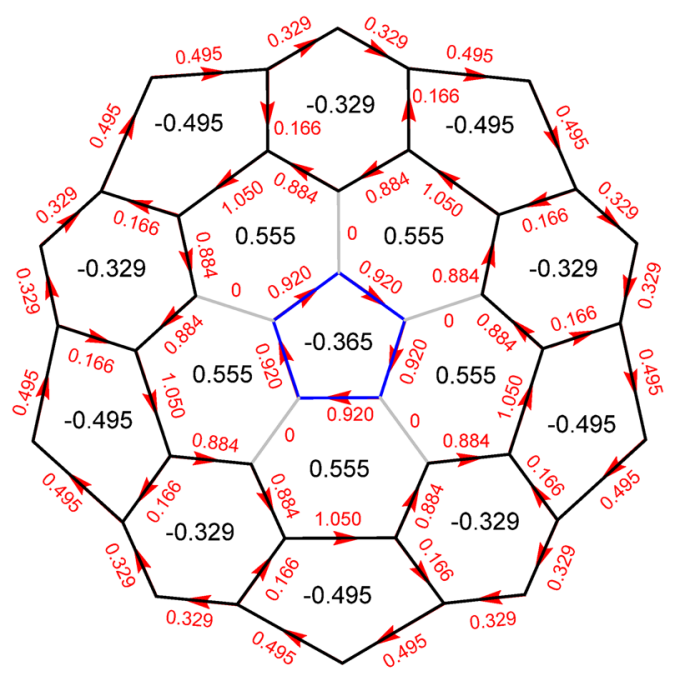

$1^{2-}$

Figure 4. Maps for the HLPM topological ring currents (in black) and the associated topological bond currents (in red) for (above) altan-corannulene (structure 1 of Figure 1; data taken from ref 5) and (below) its dianion, $\mathbf{1}^{2-}$, for comparison. The topological ring currents and bond currents are dimensionless quantities. Positive (diamagnetic) ring currents are considered to circulate counterclockwise around their respective rings, while negative (paramagnetic) ring currents flow in the clockwise sense around those rings. The various bond currents flow in the direction indicated by the arrow pointing along each bond.

these topological (that is, graph-theoretical) indices with geometrical aspects of the conjugated structure under studythe latter being, explicitly, in the form of its ring areas:

$$
\begin{aligned}
\left(\frac{J_{i}}{J_{\text {benzene }}}\right)= & 9\left\{\sum _ { ( \mu ) } \left[P_{(\mu)}+\beta \bar{\pi}_{(\mu)(\mu)} S_{(\mu)} C_{(\mu)}^{i}\right.\right. \\
& \left.+\sum_{(\mu<\nu)} \sum_{(\nu)} \beta \bar{\pi}_{(\mu)(v)}\left[S_{(\mu)} C_{(\nu)}^{i}+C_{(\mu)}^{i} S_{(\nu)}\right]\right\}
\end{aligned}
$$

In this expression, $J_{i}$ is the ring-current intensity in the $i$ th ring of the arbitrary conjugated system under study, and $J_{\text {benzene }}$ is the ring-current intensity calculated, by the same method, for benzene. $\beta$ is the standard Hückel resonance integral; $P_{(\mu)}$, as previously defined in eq 1 , is the Coulson bond order for what McWeeny called a "circuit-completing bond"17 $\mu$, and the quantities $\beta \bar{\pi}_{(\mu)(\mu)}$ and $\beta \bar{\pi}_{(\mu)(\nu)}$ are, respectively, the self- and mutual-imaginary bond-bond polarizabilities of two such bonds, $\mu$ and $\nu$, also defined earlier (in eqs 2 and 3 ). It is not necessary to go into details about the other symbols arising in eq 4 except to say the following:

(a) $S_{(\mu)}$ is a purely geometrical quantity, dependent on the ring areas. These terms can be positive or negative according to whether bonds are defined in one direction or the other.

(b) The quantities $C_{(\mu)}^{i}$-which are effectively the elements of a $(\mu \times \mu)$ matrix comprising only the entries $0,+1$, and -1 -are purely topological in nature and take on the values 0 , +1 , or -1 according to whether, respectively, (i) the $i$ th ring does not lie within the $\mu$ th cycle of the Fundamental System of Cycles $^{21,38}$ associated with the selected spanning tree, ${ }^{36}$ (ii) the $i$ th ring lies within the cycle completed by the $\mu$ th cyclecompleting bond and that cycle-completing bond is directed in the counterclockwise sense around the cycle (the $\mu$ th one) that it completes, or (iii) the ith ring lies within the $\mu$ th cycle but the $\mu$ th cycle-completing bond points in the clockwise direction around the $\mu$ th cycle.

Once it is accepted that the $\left\{S_{(\mu)}\right\}$ are purely geometrical and that the $\left\{C_{(\mu)}^{i}\right\}$ are purely topological, then, for the purpose of the discussion presented later in this Article, there is no need for any further concern, on the reader's part, about the quantities $S_{(\mu)}$ and $C_{(\mu)}^{i}$ appearing in eq 4 .

Furthermore, once the ring currents have been calculated from eq 4, bond currents are easily obtained from them by simple arithmetic. If a bond is situated in one ring only, then the bond current in that bond is numerically equal to the ring current that its ring bears, and it flows in the same sense. If a bond is shared between two rings, then the bond current in that shared bond is a result of what is effectively a competition between the two and is obtained by subtracting the (algebraical-that is, positive or negative) ring currents in the rings that share that bond. There is an exact analogy here with traditional, classical, and macroscopic Kirchhoff electrical networks: ${ }^{24,38}$ the microscopic ring currents correspond to the macroscopic loop currents, ${ }^{24}$ flowing around the several enclosed regions of the network, while the microscopic bond currents are the analogy of the currents flowing in the individual wires/arms of the macroscopic Kirchhoff network.

Calculation of London Diamagnetic Susceptibilities. Gomes and one of us have emphasized ${ }^{24}$ that if $\chi_{\text {Benzene }}^{\pi \text {-London }(\perp)}$ stands for the London contribution to the diamagnetic susceptibility of benzene, perpendicular to its (assumed) molecular plane, while $S_{\text {Benzene }}$ represents the area of a standard benzene hexagon and $J_{\text {Benzene }}$ is the ring-current intensity calculated by the same method (eq 4) for benzene, then the following simple result ${ }^{21-24}$ is obtained (eq 17 of ref 22) for an arbitrary conjugated structure with $r$ rings:

$$
\left(\frac{\chi_{\text {Structure }}^{\pi \text {-London }(\perp)}}{\chi_{\text {Benzene }}^{\pi \text {-London }(\perp)}}\right)=\sum_{\substack{\text { (rings) } \\ i=1}}^{r}\left(\frac{S_{i}}{S_{\text {Benzene }}}\right)\left(\frac{J_{i}}{J_{\text {Benzene }}}\right)
$$

where $\chi_{\text {Structure }}^{\pi \text {-Lond }(\perp)}$ is the London contribution to the magnetic susceptibility of the structure under discussion, perpendicular 
Table 1. Qualitative and Quantitative Conclusions Derived from HLPM Topological Ring-Current Calculations on 1 and 2 and Their Dianions

\begin{tabular}{|c|c|c|c|c|}
\hline structure & inner cycle (central ring) & middle cycle & outer cycle (perimeter) & $\left(\frac{\chi_{\text {Structure }}^{\pi \text {-London }(\perp)}}{\chi_{\text {Benzene }}^{\pi \text {-London }(\perp)}}\right)_{b}$ \\
\hline altan-corannulene (neutral) (1) & paramagnetic $^{a}$ & diamagnetic $^{a}$ & paramagnetic $^{a}$ & -0.8 \\
\hline altan-corannulene dianion $\left(1^{2-}\right)$ & paramagnetic $^{a}$ & diamagnetic $^{a}$ & diamagnetic $^{a}$ & 34.4 \\
\hline altan-coronene (neutral) (2) & paramagnetic & diamagnetic & paramagnetic & 5.0 \\
\hline altan-coronene dianion $\left(2^{2-}\right)$ & paramagnetic & diamagnetic & diamagnetic & 41.0 \\
\hline
\end{tabular}

${ }^{a}$ In this table, when the words "paramagnetic" or "diamagnetic" are flagged with the superscripted " $a$ " footnote, this indicates qualitative agreement with the ab initio ipso-centric calculations of Monaco and Zanasi reported in refs 2 and 4. None of the other species in this table was considered by those authors, and so no similar comparisons can be made for the systems 2 and $2^{2-}$. ${ }^{b}$ Defined in Calculation of London Diamagnetic Susceptibilities.

Table 2. Qualitative and Quantitative Conclusions Derived from HLPM Topological Ring-Current Calculations on Double Altans 3 and 4 and Their Dianions

\begin{tabular}{|c|c|c|c|c|c|}
\hline structure & inner cycle (central ring) & first middle cycle & second middle cycle & outer cycle (perimeter) & $\left(\frac{\chi_{\text {Structure }}^{\pi \text {-London }(\perp)}}{\chi_{\text {Benzene }}^{\pi \text {-London }(\perp)}}\right)_{a}$ \\
\hline$(\text { altan })^{2}$-corannulene (neutral) $(3)$ & paramagnetic & diamagnetic & paramagnetic & diamagnetic & 24.0 \\
\hline$(\text { altan })^{2}$-corannulene dianion $\left(3^{2-}\right)$ & paramagnetic & diamagnetic & paramagnetic & diamagnetic & 24.0 \\
\hline$(\text { altan })^{2}$ - coronene (neutral) $(4)$ & paramagnetic & diamagnetic & paramagnetic & diamagnetic & 26.0 \\
\hline$(\text { altan })^{2}-$ coronene dianion $\left(4^{2-}\right)$ & paramagnetic & diamagnetic & paramagnetic & diamagnetic & 26.0 \\
\hline
\end{tabular}

Table 3. Qualitative and Quantitative Conclusions Derived from HLPM Topological Ring-Current Calculations on Triple Altans 5 and 6 and Their Dianions

\begin{tabular}{|c|c|c|c|c|c|c|}
\hline structure & $\begin{array}{l}\text { inner cycle (central } \\
\text { ring) }\end{array}$ & $\begin{array}{l}\text { first middle } \\
\text { cycle }\end{array}$ & $\begin{array}{l}\text { second middle } \\
\text { cycle }\end{array}$ & $\begin{array}{l}\text { third middle } \\
\text { cycle }\end{array}$ & $\begin{array}{l}\text { outer cycle } \\
\text { (perimeter) }\end{array}$ & $\left(\frac{\chi_{\text {Structure }}^{\pi \text {-London }(\perp)}}{\chi_{\text {Benzene }}^{\pi \text {-London }(\perp)}}\right)_{a}$ \\
\hline$(\text { altan })^{3}$-corannulene (neutral) (5) & paramagnetic & diamagnetic & paramagnetic & paramagnetic & diamagnetic & 16.1 \\
\hline $\begin{array}{l}(\text { altan })^{3} \text {-corannulene dianion } \\
\left(\mathbf{5}^{2-}\right)\end{array}$ & paramagnetic & diamagnetic & paramagnetic & paramagnetic & diamagnetic & 16.1 \\
\hline$(\text { altan })^{3}$ - coronene (neutral) (6) & diamagnetic & diamagnetic & paramagnetic & diamagnetic & diamagnetic & 40.2 \\
\hline$(\text { altan })^{3}$ - coronene dianion $\left(6^{2-}\right)$ & diamagnetic & diamagnetic & paramagnetic & diamagnetic & diamagnetic & 40.2 \\
\hline
\end{tabular}

to its assumed molecular plane, ${ }^{21,22} \chi_{\text {Benzene }}^{\pi \text {-London }(\perp)}$ is the analogous quantity for benzene, $S_{i}$ is the area of the $i$ th ring, and $J_{i}$ is the ring-current intensity in the $i$ th ring, calculated from eq 4. The quantity $\left(\frac{\chi_{\text {Structure }}^{\pi \text {-London }(\perp)}}{\chi_{\text {Benzene }}^{\pi-\operatorname{Lond}(\perp)}}\right)$ is what was originally computed (by London ${ }^{15}$ ) in the first quantum-mechanical ring-current calculations, in the 1930s-although without the intermediate mention, nor the explicit calculation, of ringcurrent intensities per se.

The numerical values of $\left(\frac{\chi_{S \text { trunture }}^{\pi \text {-Lond }(\perp)}}{\chi_{\text {Benzene }}^{\pi \text {-Lond }}(\perp)}\right)$ for structures 1-6 and their dianions are given in the right-hand columns of Tables 1 , 2 , and 3. Of these, only one (1) has a negative value for $\left(\frac{\chi_{\text {Strondure }}^{\pi \text {-Lond }}(\perp)}{\chi_{\text {Benzene }}^{\pi \text {-Lond }(\perp)}}\right)$, which (by a naive-but, in practice, unwarranted $^{39}$ - extension from monocyclic to polycyclic systems) might intuitively ${ }^{39-44}$ be thought of as indicating antiaromaticity-but please see ref 44 for further critical comment on this kind of reasoning. All of the others have positive values for this index, and those with the greatest values (of ca. 40) are $2^{2-}, 6$, and $6^{2-}$.

It may be mentioned in passing that, in the case of conjugated systems consisting only of benzenoid rings, all the factors $\left(\frac{S_{i}}{S_{\text {Benzene }}}\right)$ are taken to be identically equal to 1 and so, as a special case, the quantity $\left(\frac{\chi_{S_{\text {tsuructure }}}^{\pi \text {-London }(\perp)}}{\chi_{\text {Benzene }}^{\pi \text {-Lond }}(\perp)}\right)$ is seen numerically to be equivalent to the index invoked in ref 28 (and called there " $\Sigma J(\mathrm{CD}-\mathrm{HL})$ "), which is evaluated by simply adding all of the ring-current intensities (expressed as a ratio to the benzene value) in a given conjugated structure, which, by assumption in this special case, necessarily has no rings other than benzenoid ones, each being of the standard (benzene) area.

Numerical Accuracy of Reported Calculations. It is our custom always to compute in double precision and to quote the results of HLPM calculations to three decimal places. This policy becomes less justifiable when the size of the structures increases as there is then more opportunity for round-off error. In the case of the calculations on the double ( 3 and 4 ) and triple (5 and 6) altans studied here-structures that involve up to 43 rings-we also experimented with basing the calculations on two or more different spanning trees ${ }^{21-24,35,36}$ of the molecular graph under consideration. A special kind of spanning tree that is unbranched ${ }^{36}$ was initially prescribed, in his classic paper, ${ }^{17}$ by McWeeny, who called such spanning trees open chains. ${ }^{36}$ Later, Gayoso and Boucekkine ${ }^{35}$ extended McWeeny's formalism ${ }^{17}$ to encompass branched ${ }^{36}$ spanning 
trees. Our experimentation showed that basing a calculation on a branched spanning tree seems to reduce the opportunity for round-off errors-ostensibly because each ring of the conjugated system under study then generally appears in fewer terms (during the intermediate calculation) than is the case when an open chain ${ }^{17,36}$ is adopted as the starting point. As a result of these investigations, we feel justified in reporting ring currents and bond currents in mono-altans ( 1 and 2 ) to three decimal places (as usual) but we quote these quantities for the double (3 and 4) and triple altans (5 and 6) to only two decimal places.

\section{RESULTS AND PRELIMINARY DISCUSSION}

Patterns of HLPM Bond Currents in the Various Concentric Cycles within Altan-Corannulene (1) and $\mathbf{1}^{2-}$. The HLPM ring-current and bond-current maps for neutral altan-corannulene (1) (taken from Figure 4 of ref 5), and the corresponding maps for $\mathbf{1}^{2-}$, are depicted in Figure 4. Note that in these structures the current in the central (fivemembered) ring (emphasized in blue in Figure 4) is essentially isolated $\left(\right.$ decoupled $^{45}$ ) from the rest of the system by spokes ${ }^{45}$ bonds that, by symmetry, bear zero bond currents (and which are depicted gray in color in Figure 4). It is crucial to observe that the bond currents around the peripheries of $\mathbf{1}$ and $\mathbf{1}^{2-}$ are very different-both in sign and in their paramagnetic/ diamagnetic character-while the bond currents in the corresponding internal bonds of $\mathbf{1}$ and $\mathbf{1}^{2-}$ are identical (to within a digit in the third decimal place, the accuracy to which numerical results are quoted). Ring currents in $\mathbf{1}$ and $\mathbf{1}^{2-}$ are thus very different, despite the fact that bond currents in corresponding internal bonds are identical in the two species.

It is seen from Table 1 that the qualitative diamagnetic/ paramagnetic nature of the bond-current circulations around the several concentric cycles within 1 and $\mathbf{1}^{2-}$ - predicted by the HLPM calculations, going from the inner cycle (the central ring) to the outer cycle (the perimeter) - is entirely in accord with what is predicted by the ipso-centric ab initio approach and reported by Monaco, Zanasi, and Memoli in refs 2 and 4. That pattern is (outward, from the center) as follows: paramagnetic/diamagnetic/paramagnetic (for the neutral species (1)) and paramagnetic/diamagnetic/diamagnetic (for the dianion $\left.\left(\mathbf{1}^{2-}\right)\right)$.

Of the two species altan-corannulene (1) and its dianion $\left(1^{2-}\right)$, it is the dianion that has the higher value (ca. 34) of the quantity $\left(\frac{\chi_{\text {Structure }}^{\pi \text {-London }(\perp)}}{\chi_{\text {Benzene }}^{\pi \text {-London }(\perp)}}\right)$.

The reader is asked to note two very important observations to be drawn from Figure 4:

(i) The bond currents around the perimeter are very different in 1 and $\mathbf{1}^{2-}$, alternating between 0.329 and 0.495 in the paramagnetic (clockwise) direction for $\mathbf{1}$ and between 2.020 and 2.186 in the diamagnetic (counterclockwise) sense for $\mathbf{1}^{2-}$. Such a contrast is perhaps entirely to be expected, in view of the necessity for the notional addition of two extra $\pi$ electrons as the transition is made from the neutral species (1) to its dianion $\left(\mathbf{1}^{2-}\right)$.

(ii) However, despite the fact that the ring currents in $\mathbf{1}$ are very different from those in $\mathbf{1}^{2-}$, it is seen by careful scrutiny of the upper and lower parts of Figure 4 that the bond currents in every one of the corresponding internal (that is, nonperipheral) bonds of $\mathbf{1}$ and $\mathbf{1}^{2-}$ are all precisely the same (to within one digit in the third decimal place, the accuracy to which ring currents and bond currents are quoted here). Only bond currents in bonds around the perimeter are different in $\mathbf{1}$ and $\mathbf{1}^{2-}$.

Later, we shall comment on and attempt to explain these phenomena, in the section Rationalization of Bond-Current Patterns in Single and Iterated Altans and Their Dianions.

Patterns of HLPM Bond Currents in the Various Concentric Cycles within Altan-Coronene (2) and $2^{2-}$. The HLPM ring-current and bond-current maps for (above) neutral altan-coronene (2) (taken from ref 5) and (below) the corresponding maps for $\mathbf{2}^{2-}$ are depicted in Figure 5. It can again be noted that, in $\mathbf{2}$ and $\mathbf{2}^{2-}$, the current in the central ring (which is outlined in blue, for emphasis, in Figure 5) is essentially isolated (decoupled ${ }^{45}$ ) from the rest of the system by spokes ${ }^{45}$ bonds that, by symmetry, bear zero bond currents (and which are depicted gray in color in Figure 5). It is vital

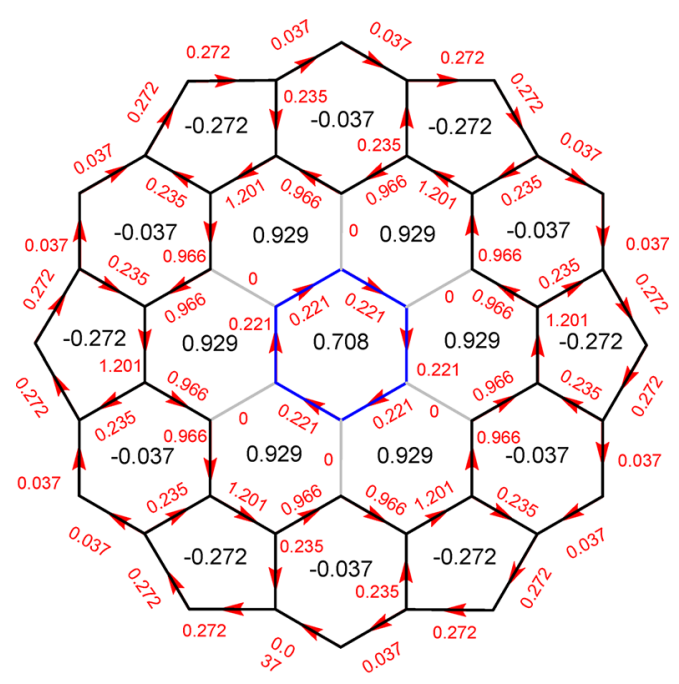

2

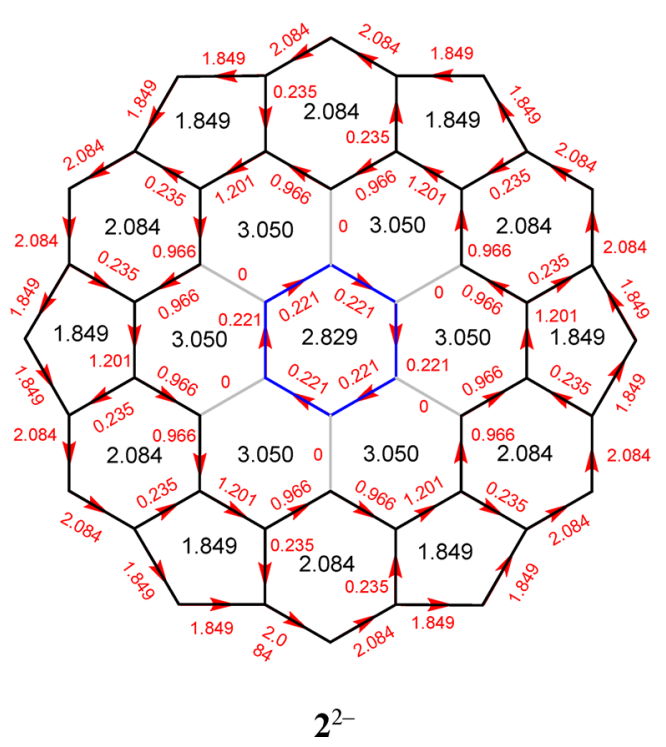

Figure 5. Maps for the HLPM topological ring currents (in black) and the associated topological bond currents (in red) for (above) altan-coronene (structure 2, Figure 1; taken from ref 5) and (below) its dianion, $\mathbf{2}^{2-}$, for comparison. For the conventions on displaying topological ring currents and bond currents, please see the caption to Figure 4. 
once again for our purposes to observe that, while the ring currents in 2 and $2^{2-}$ and the bond currents in bonds around the peripheries of $\mathbf{2}$ and $\mathbf{2}^{2-}$ are very different, the bond currents in the corresponding internal bonds-that is, those not on the perimeter-in $\mathbf{2}$ and $\mathbf{2}^{2-}$ are identical, to the accuracy quoted. This is despite the fact that ring currents in $\mathbf{2}$ and $2^{2-}$ are entirely different.

Table 1 (second half) shows that the qualitative diamagnetic/paramagnetic nature of the bond-current circulations around the several concentric cycles in 2 and $\mathbf{2}^{2-}-$ from the inner cycle (the central ring) to the outer cycle (the perimeter) - predicted by these HLPM calculatons is entirely the same as for the corresponding members of the series $\mathbf{1}$ and $\mathbf{1}^{2-}$, reported in the first half of Table 1 . That is (from the inner cycle outward): paramagnetic/diamagnetic/paramagnetic (for the neutral species (2)) and paramagnetic/ diamagnetic/diamagnetic (for the dianion $\left(2^{2-}\right)$ ).

On this occasion, however, no comparison with ab initio calculations is available from ref 4 , as was the case with $\mathbf{1}$ and $\mathbf{1}^{2-}$. As with $\mathbf{1}$ and $\mathbf{1}^{2-}$, however, of the two species $\left(\mathbf{2}\right.$ and $\left.\mathbf{2}^{2-}\right)$ related to altan-coronene that are considered here, the dianion $\left(2^{2-}\right)$, as before, has the higher value of $\left(\frac{\chi_{\text {Strundure }}^{\pi \text {-London }(\perp)}}{\chi_{\text {Benzene }}^{\pi \text {-Lond }}(\perp)}\right)$ (ca. 41). This observation is not inconsistent with the fact that the dianion $\left(2^{2-}\right)$ has a preponderance of diamagnetic cycles (two, out of three), whereas 2 (with a $\left(\frac{\chi_{\text {Structure }}^{\pi \text {-London }(\perp)}}{\chi_{\text {Benzene }}^{\pi \text {-London }(\perp)}}\right)$ value of only $\sim 5$ ) hosts two paramagnetic circulations and only a single diamagnetic one.

Once more, we note two important observations drawn from Figure 5:

(i) The bond currents around the respective perimeters are again very different in $\mathbf{2}$ and in $\mathbf{2}^{2-}$, being weakly in the paramagnetic direction for 2 (alternating between about 0.04 and 0.27 ) and strongly in the diamagnetic sense (between about 1.85 and 2.08 ) for $2^{2-}$.

(ii) However, it is again seen by close inspection of both parts of Figure 5 that, despite the fact that the ring currents overall in $\mathbf{2}$ are very different from those in $\mathbf{2}^{2-}$, the bond currents in the corresponding internal bonds of $\mathbf{2}$ and $\mathbf{2}^{2-}$ are all precisely the same (to within a digit in the third decimal place, the accuracy to which ring currents and bond currents are being quoted here). Only bond currents in bonds around the perimeter are different in $\mathbf{2}$ and $\mathbf{2}^{2-}$. We shall likewise comment on and attempt to explain these phenomena later in this Article, in the section Rationalization of Bond-Current Patterns in Single and Iterated Altans and Their Dianions.

Patterns of HLPM Bond Currents in the Various Concentric Cycles within (Altan) ${ }^{2}$-Corannulene (3) and $3^{2-}$. The HLPM ring-current and bond-current maps for neutral (altan) $)^{2}$-corannulene (3) (which, it should be noted, are precisely the same as those for its dianion $\left(3^{2-}\right)$ ) are depicted in Figure 6. In the case of this double altan, it is not just those currents in corresponding bonds in the interior of the structures that are identical in the neutral species (3) and the dianion $\left(3^{2-}\right)$, but the currents in the bonds around the peripheries of 3 and $3^{2-}$ are also identical-because the ringcurrent intensities in the corresponding rings of (3) and $3^{2-}$ are exactly the same. This result initially surprised us to the extent that we felt obliged independently to repeat the calculation-running it in all three times-lest the outputs might somehow have become interchanged. In the course of

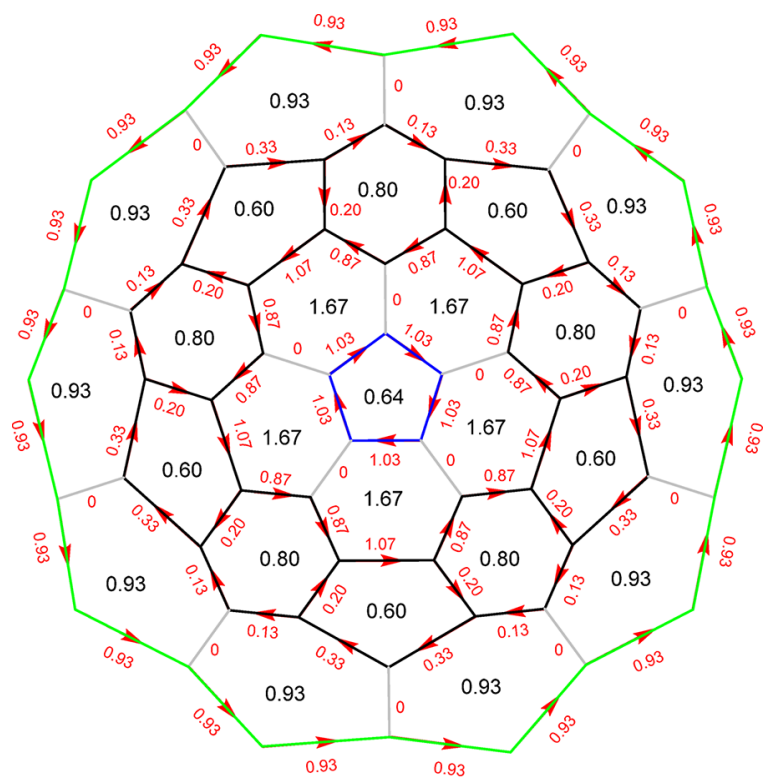

$3 \& 3^{2-}$

Figure 6. Maps for the topological ring currents (in black) and the associated topological bond currents (in red) for neutral (altan $)^{2}$ corannulene $(3)$ and for its dianion $\left(3^{2-}\right)$. Note that the ring-current and bond-current maps for the dianion $\left(3^{2-}\right)$ of 3 are identical with those of neutral 3 itself: please see the text for a discussion of this phenomenon. The diatropic [4n]-perimeter ${ }^{25}$ of this iterated altan ${ }^{25}$ should also be noted in view of the comments of Monaco-based on ab initio calculations-in ref 25 .

this, we also took the opportunity additionally to vary the spanning trees ${ }^{21-24,35,36}$ on which these calculations were based. Each time, however, the same result emerged: the ring currents and the corresponding respective bond currents in all rings in 3 and $3^{2-}$ are identical. Possible reasons for this are discussed later in this Article, in the section Rationalization of Bond-Current Patterns in Single and Iterated Altans and Their Dianions.

It can again be noted from Figure 6 that in 3 and $3^{2-}$, not only the current flowing around the bonds in the central (fivemembered) ring (emphasized in blue in Figure 6) but also the current in the outer perimeter (emphasized in green) are essentially isolated (decoupled ${ }^{45}$ ) from the rest of the system by spokes $^{45}$ bonds-on this occasion, two sets of themwhich, by symmetry, bear zero bond currents; (these bonds are depicted in gray in Figure 6). These incisions by current-free spokes bonds also result in the isolation of the second and third cycles fom the center (depicted in black in Figure 6), which therefore effectively form an independent system of their own.

The first half of Table 2 indicates that, as was the case for altan-corannulene (1) and altan-coronene (2), the patterns of current flow around the various concentric cycles in altan ${ }^{2}$ coranulene ( 3 ) and in $3^{2-}$ alternate in nature, as one goes from the inner cycle (the central ring) to the outer cycle (the perimeter) - that is, for both 3 and $3^{2-}$, the pattern is (from the inner ring, going outward) as follows: paramagnetic/ diamagnetic/paramagnetic/diamagnetic.

There are no ab initio data in refs 2 and 4 to compare with this but recall that, in the case of $\mathbf{1}$ and $\mathbf{1}^{2-}$, the analogous patterns predicted by the ab initio ispso-centric approach and 
the HLPM formalism agreed qualitatively. The value for $\left(\frac{\chi_{\text {Structure }}^{\pi}}{\chi_{\text {Benzene }}^{\pi \text {-Lond }(\perp)}}\right)$ for 3 and $3^{2-}$ is $\sim 24$.

Patterns of HLPM Bond Currents in the Various Concentric Cycles within (Altan) $)^{2}$-Coronene (4) and $4^{2-}$. The HLPM ring-current and bond-current maps for neutral (altan) $)^{2}$-coronene (4) (which is the same for its dianion $\left(4^{2-}\right)$ ) are shown in Figure 7. As was the case for the neutral species

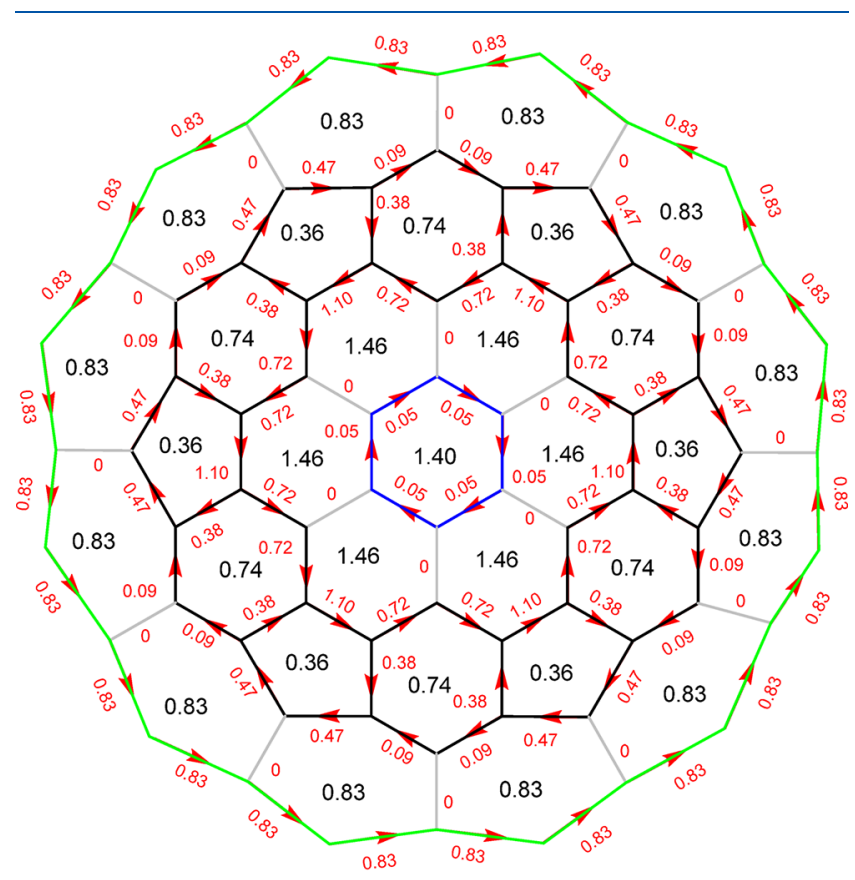

$4 \& 4^{2-}$

Figure 7. Maps for the HLPM topological ring currents (in black) and the associated topological bond currents (in red) for neutral $(\text { altan })^{2}$-coronene (4), which are identical to the corresponding maps for its dianion $\left(4^{2-}\right)$. In view of the remarks of Monaco in ref 25which were based on ab initio calculations-it should also be noted that this iterated altan has a diatropic perimeter ${ }^{16}$ despite, as with all iterated altans, ${ }^{25}$ that perimeter's being of the $[4 n]$ type.

and the dianion of the double altan of corannulene ( 3 and $3^{2-}$ ), it was found-despite rigorous cross-checking such as was described earlier for the calculations on 3 and $3^{2-}$-that not just those currents in the corresponding respective bonds in the interior of the structures are identical in the neutral species (4) and in the dianion $\left(4^{2-}\right)$, but the currents in the bonds around the peripheries of 4 and $4^{2-}$ are identical also. Possible reasons for this are discussed later in this Article, in the section Rationalization of Bond-Current Patterns in Single and Iterated Altans and Their Dianions. As was the case with 3 and $3^{2-}$, for 4 and $4^{2-}$ not only the current in the central ring (emphasized in blue in Figure 7) but also the current in the outer perimeter (emphasized in green) are essentially isolated (decoupled $^{45}$ ) from the rest of the system by spokes ${ }^{45}$ bonds-here, once again, two sets of them-which, by symmetry, bear zero bond currents. The system represented by the second and third cycles (from the center), depicted in black in Figure 4, is also isolated by these spokes bonds (which are themselves depicted in gray in Figure 7) and effectively exists independently from the rest of the structure.
The lower half of Table 2 indicates that the current flow around the various concentric cycles in altan ${ }^{2}$-coronene (4) and in $4^{2-}$ once again alternate in nature, as one goes from the inner cycle (the central ring) to the outer cycle (the perimeter), in exactly the same manner as was observed in the case of 3 and $3^{2-}$. The pattern for 4 and $4^{2-}$ is as follows: paramagnetic/diamagnetic/paramagnetic/diamagnetic.

The value of $\left(\frac{\chi_{\text {Structure }}^{\pi \text {-London }(\perp)}}{\chi_{\text {Benzene }}^{\pi-\operatorname{Lond}(\perp)}}\right)$ for 4 and $4^{2-}$ is $\sim 24$.

Patterns of HLPM Bond Currents in the Various Concentric Cycles within (Altan) ${ }^{3}$-Corannulene (5) and $5^{2-}$. The HLPM ring-current and bond-current maps for neutral (altan) ${ }^{3}$-corannulene (5) (which is the same for its dianion $\left(\mathbf{5}^{2-}\right)$ ) are shown in Figure 8 . The by-now expected pattern emerges that, once again, neutral species (5) and dianion $\left(5^{2}\right)$ have identical ring currents in their corresponding rings and, as an inevitable consequence, identical bond currents both for their respective peripheral bonds and for their corresponding internal bonds. Possible reasons for this are discussed later in this Article in the section Rationalization of Bond-Current Patterns in Single and Iterated Altans and Their Dianions. Once again, certain cycles and families of cycles are essentially isolated (decoupled ${ }^{45}$ ) from the rest of the system by spokes ${ }^{45}$ bonds that, by symmetry, bear zero bond currents (and which are shown in gray in Figure 8). In the case of $\mathbf{5}$ and $\mathbf{5}^{2-}$, not only is the current in the (fivemembered) central ring (emphasized in blue in Figure 8) isolated in this way but the perimeter and the penultimate cycle (emphasized in green in Figure 8) effectively form an independent system of their own, being decoupled from the inner three cycles by current-free spokes bonds separating the third and fourth cycles from the center (as depicted in Figure 8 ). These incisions by several families of spokes bonds also isolate the second and third cycles from the circulations occurring in the central ring and from the current flow in the fourth and fifth cycles; these cycles (depicted in black in Figure 8) do, thereby, likewise form what is essentially an independent system of their own.

The first half of Table 3 indicates that the flows of current around the various concentric cycles in (altan $)^{3}$-coroannulene (5) and in $5^{2-}$ break the previous pattern of alternation that was observed for the single (1 and 2 ) and double (3 and 4) altans and their dianions. The bond-current pattern-going from the central ring outward to the perimeter-for 5 and $5^{2-}$ is as follows: paramagnetic/diamagnetic/paramagnetic/paramagnetic/diamagnetic.

There is a preponderance of paramagnetic cycles, and this is consistent with a somewhat low $\left(\frac{\chi_{S_{\text {truncure }}}^{\pi-\text { London }(\perp)}}{\chi_{\text {Benzene }}^{\text {-Lind }}(\perp)}\right)$ ratio of only $\sim 16$.

Patterns of HLPM Bond Currents in the Various Concentric Cycles within (Altan) ${ }^{3}$-Coronene (6) and $6^{2-}$. Finally, the HLPM ring-current and bond-current maps for neutral (altan) $)^{3}$-coronene (6) (which are the same as for its dianion $\left(6^{2-}\right)$ ) are shown in Figure 9. The pattern, by now expected, is again encountered-namely, that once more the neutral species (this time 6) and the dianion of it $\left(6^{2-}\right)$ have identical ring currents in all of their corresponding rings andtherefore, as an inevitable consequence-they have identical bond currents both in their respective peripheral bonds and in their internal bonds. Possible reasons for this are discussed later in this Article in the section Rationalization of BondCurrent Patterns in Single and Iterated Altans and Their 


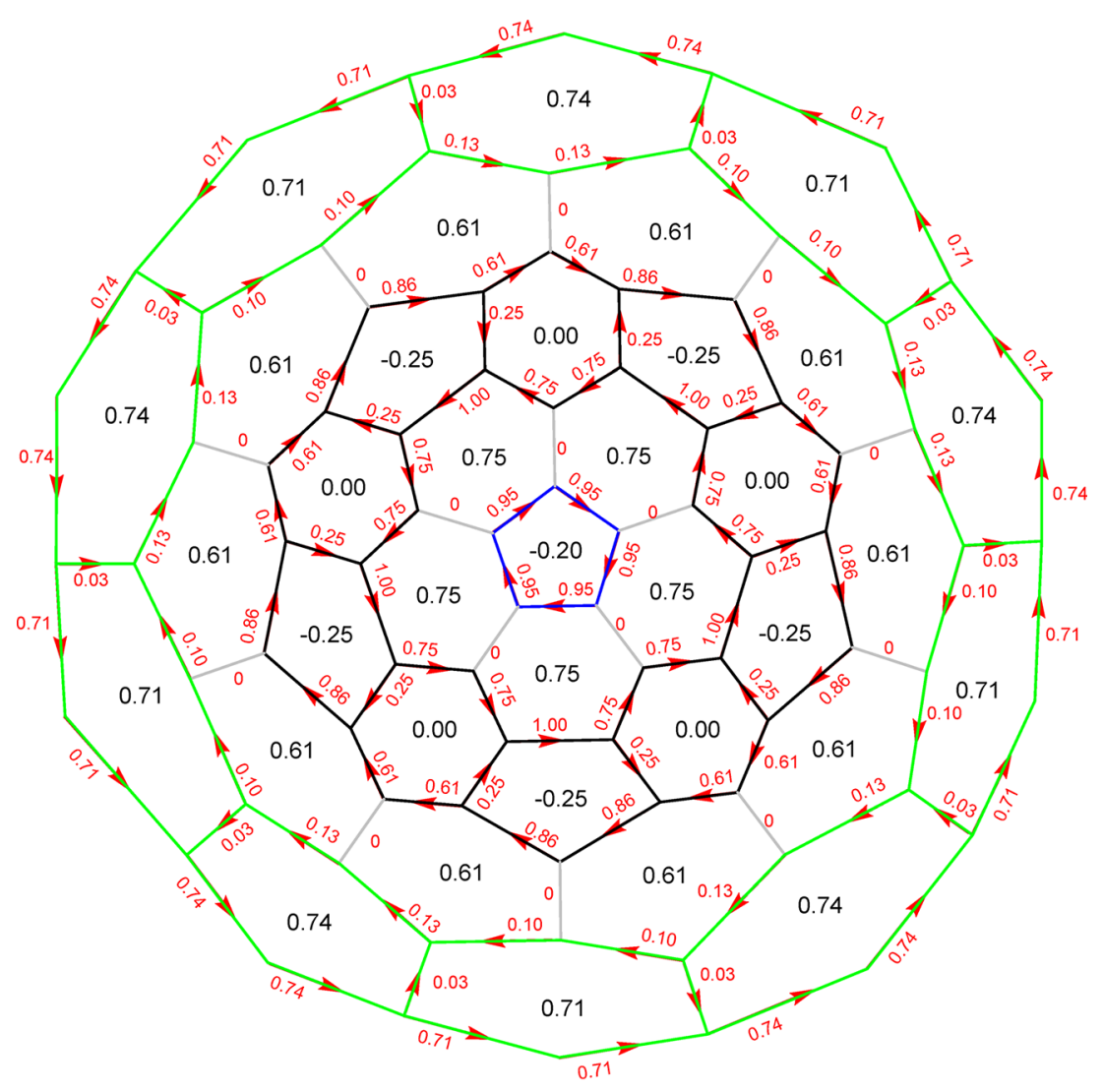

$5 \& 5^{2-}$

Figure 8. Maps for the HLPM topological ring currents (in black) and the associated topological bond currents (in red) for neutral (altan) ${ }^{3}$ corannulene (5), which are identical to the corresponding maps for its dianion $\left(5^{2-}\right)$. It should also be noted that this iterated altan has a diatropic perimeter, ${ }^{25}$ notwithstanding the fact that, like all iterated altans, ${ }^{25}$ its perimeter is of the $[4 n]$ type. ${ }^{25}$

Dianions. As in $\mathbf{5}$ and $\mathbf{5}^{2-}$ the current in the central ring of $\mathbf{6}$ and $\mathbf{6}^{2-}$ (emphasized in blue in Figure 9) is isolated from the rest of the structure by spokes bonds bearing zero bond current (shown in gray in Figure 9), and the perimeter and the penultimate cycle (emphasized in green in Figure 8) -also as was found for $\mathbf{5}$ and $\mathbf{5}^{2-}$-effectively form an independent system of their own, being decoupled from the inner three cycles by current-free spokes ${ }^{45}$ bonds separating the third and fourth cycles from the center (as depicted in Figure 9). As with 5 and $5^{2-}$, this likewise leaves the second and third cycles (represented in black in Figure 9) isolated, to form their own independent system.

The lower half of Table 3 indicates that the flow of current around the various cycles in altan $^{3}$-coronene $(6)$ and in $6^{2-}$ also break the previous pattern of alternation that was initially observed for the single (1 and 2) and double ( 3 and 4 ) altans and their dianions. However, the pattern for this triple altan $\left((\text { altan })^{3}\right.$-coronene $\left.(6)\right)$ and its dianion $\left(6^{2-}\right)$ is different from that observed in the case of the other triple altans studied here $\left((\text { altan })^{3}\right.$-corannulene $(5)$, and its dianion $\left.\left(5^{2-}\right)\right)$. The bondcurrent pattern for $\mathbf{6}$ and $\mathbf{6}^{2-}$ is (from the central ring, going outward to the perimeter) as follows: diamagnetic/diamagnetic/paramagnetic/diamagnetic/diamagnetic.

It might be noted that (altan $)^{3}$-corannulene $\left(5\right.$ and $5^{2-}$ ) has a preponderance of paramagnetic cycles (three out of five) while (altan $)^{3}$-coronene $\left(6\right.$ and $6^{2-}$ ) has more diamagnetic cycles (four of them) than paramagnetic cycles (one). This is

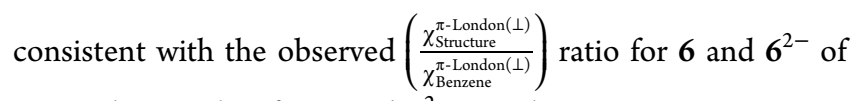
$\sim 40$, whereas that for 5 and $\mathbf{5}^{2-}$ is only ca. 16 .

\section{RATIONALIZATION OF BOND-CURRENT PATTERNS IN SINGLE AND ITERATED ALTANS AND THEIR DIANIONS}

In this section, we attempt to rationalize the following observations that have so far been made in this Article:

(i) In the mono-altans $\mathbf{1}$ and 2, the bond currents in the bonds around the perimeter are different in the dianion from in the neutral species, but, despite very different ring currents in the neutral species $\mathbf{1}$ and $\mathbf{2}$ and their respective dianions, the bond currents in all the corresponding internal bonds are the same for both the neutral species and the dianion, for both 1 and 2.

(ii) In the iterated altans - both double (3 and 4) and triple (5 and 6) -the bond currents in all bonds (both internal and peripheral) in the dianions are identical to those in the corresponding bonds of the respective neutral species.

These unexpected and surprising observations are discussed and rationalized in the next three subsections.

Configuration of the HOMO-LUMOs in the MonoAltans (1 and 2) and Iterated Altans (4-6). The disposition of the energy levels in the two neutral monoaltans ( 1 and 2 ) and the pairs of neutral double ( 3 and 4$)$ and triple (5 and $\mathbf{6})$ altans, as well as their respective dianions, are 


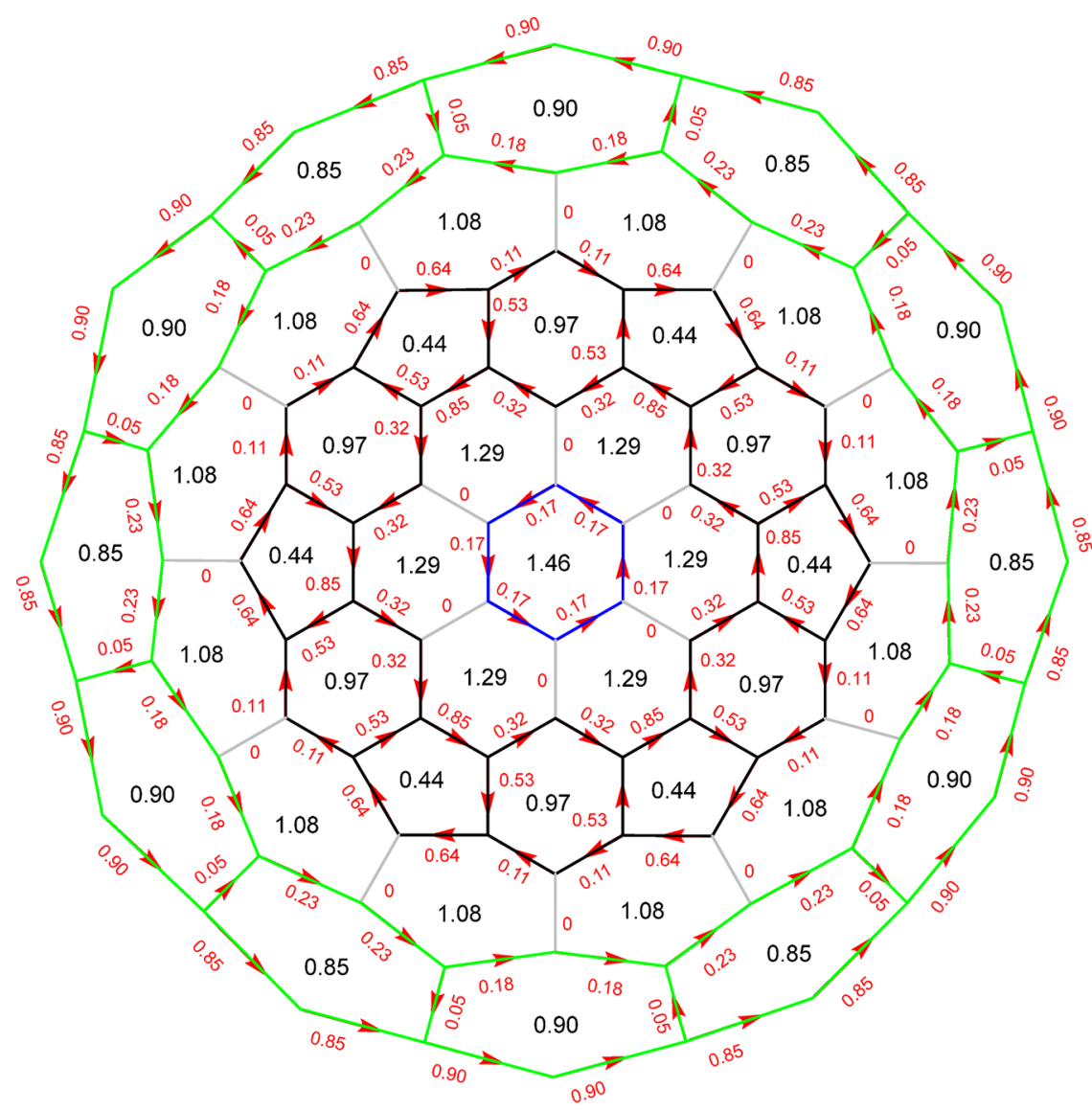

$6 \& 6^{2-}$

Figure 9. Maps for the HLPM topological ring currents (in black) and the associated topological bond currents (in red) for neutral (altan) ${ }^{3}$ coronene (6), which (as was the case with 3,4 , and 5 ) are the same as the corresponding maps for its dianion $\left(6^{2-}\right)$. It should also be noted that this iterated altan likewise has a diatropic perimeter ${ }^{16}$ despite the fact that, as with all iterated altans, ${ }^{25}$ that perimeter is (by definition) of the [4n] type. $^{25}$

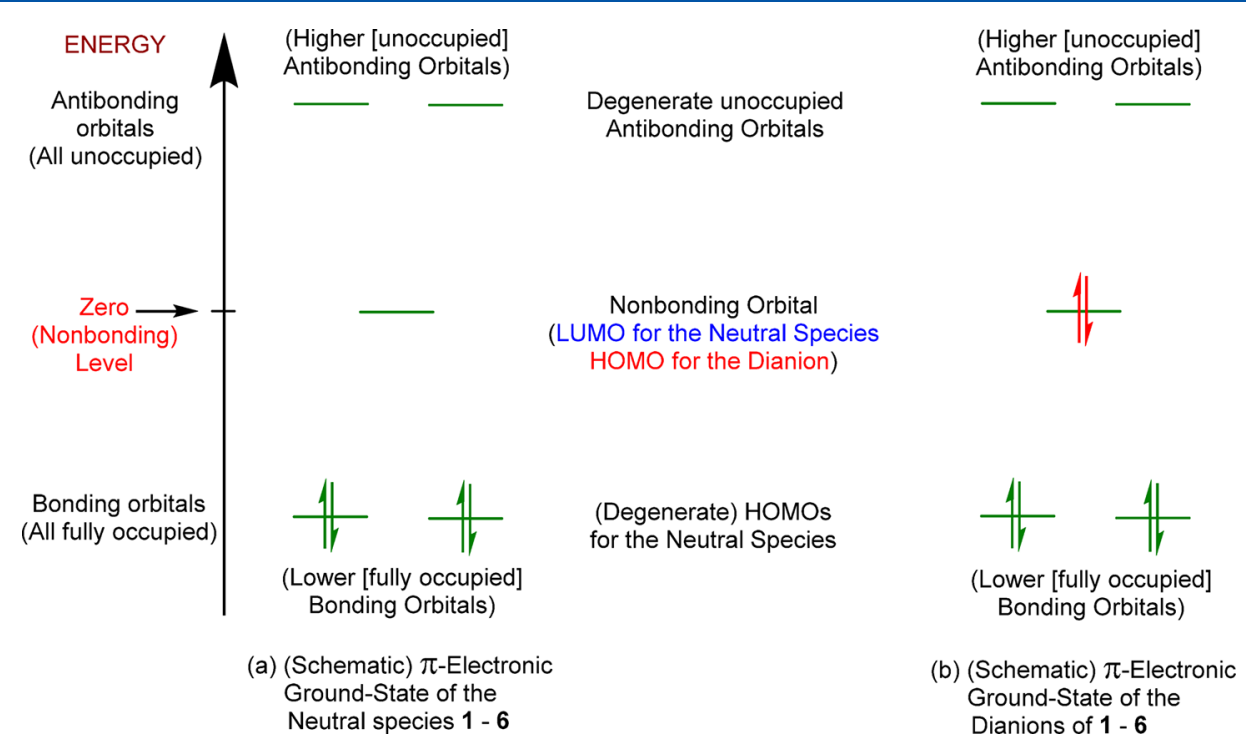

Figure 10. Schematic respresentation of the $\pi$-electronic ground-state configuration, obtained from an application of the Aufbau process, for (a) the neutral species 1-6 and (b) the dianions of 1-6. The extra two electrons that are possessed by each of the dianions of 1-6 are denoted in red on the right-hand side of the figure; they are both assigned, by the Aufbau process, to occupy the lone, nonbonding orbital (with paired spins).

schematically illustrated in Figure 10. The following important features should be noted: (i) The highest-occupied molecular orbitals (HOMOs) in the neutral species of $\mathbf{1 - 6}$ are a pair of degenerate, doubly 
occupied bonding orbitals. (Please see the left-hand side of Figure 10, colored in green.)

(ii) The lowest-unoccupied molecular orbital (LUMO) in the neutral species is a lone (nondegenerate) nonbonding orbital. In graph-theoretical terms, it is a single (nonrepeated) zero eigenvalue of the associated molecular graph. ${ }^{14,20}$ (Please see the left-hand side of Figure 10.)

(iii) The two antibonding orbitals immediately above the nonbonding orbital in 1-6 are degenerate and-like all of the other antibonding orbitals above them-they are unoccupied in both the neutral species and in the dianion. (Please see parts (a) and (b) of Figure 10.)

(iv) When, in order to make the transition from the neutral species (of 1-6) to the corresponding dianion, two extra $\pi$ electrons are notionally added according to the provisions of the Aufbau principle, ${ }^{14,37}$ these two extra $\pi$-electrons go, with paired spins, into the lone, nonbonding orbital (as shown in red on the right-hand side of Figure 10). The single zeroenergy level, by accommodating the extra two $\pi$-electrons in this way, thereby goes from being the LUMO of the neutral species to being the (fully occupied) HOMO of the dianion.

(v) Now, it is well-known that, in any arbitrary conjugated system, the sum of the coefficients of a nonbonding MO on all carbon atoms adjacent to a given carbon atom is zero-see, for example, page 100 of ref 14 .

(vi) In addition to obeying property (v), each of the unique nonbonding orbitals in structures $\mathbf{1 - 6}$ is such that its only nonzero coefficients alternate around the periphery, as is shown, for example, in the case of $\mathbf{1}$ in Figure 11. The

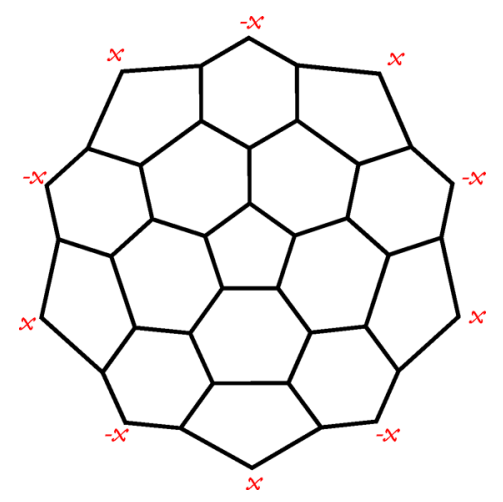

Figure 11. Nonzero coefficients (marked $x$ or $-x$ ) of the nonbonding $\mathrm{MO}$ in altan-corannulene (1). All unmarked centers have a zero coefficient in the nonbonding MO. For a normalized MO, $x=1 / \sqrt{10}$.

nonbonding MO for one of the triple altans (6) is illustrated in Figure 12. Although they are not explicitly displayed here, similar patterns are also encountered for the nonbonding orbitals of $\mathbf{2 - 5}$.

Configuration of the HOMO-LUMOs in the MonoAltans of Other Species. We temporarily leave the domain of the altans of corannulene $(\mathbf{1}, 3$, and 5$)$ and coronene $(\mathbf{2}, \mathbf{4}$, and 6) because, at this stage of the investigation, we consulted the archives of our previously published calculations ${ }^{6,7,22}$ on the mono-altans of 10 other species - those of benzene $(7),{ }^{22}$ naphthalene $(8){ }^{6}$ pyrene $(9){ }^{6}$ perylene $(10){ }^{7}$ peropyrene (11), ${ }^{7}$ kekulene (12), ${ }^{6}$ azulene (13), ${ }^{6}$ acenaphthylene (14), and fluoranthene $(\mathbf{1 5})$; $^{7}$ we also studied pyracylene $(\mathbf{1 6}),{ }^{6}$ but that is excluded from consideration for the moment, for reasons that will become apparent. (Structures 7-16 are illustrated in refs 6, 7, and 22.) The neutral altans of all the

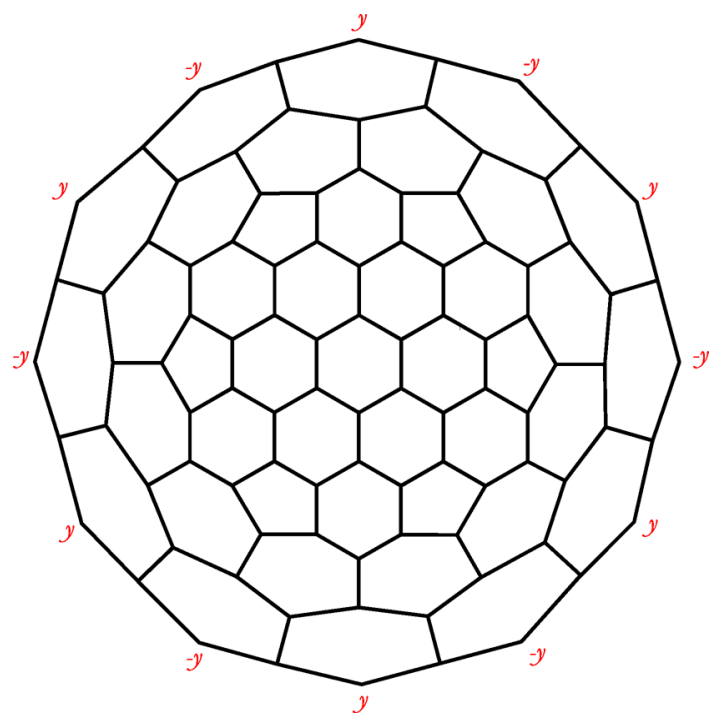

Figure 12. Nonzero coefficients (marked $y$ or $-y$ ) of the nonbonding $\mathrm{MO}$ in $(\text { altan })^{3}$-coronene (6). All unmarked centers have a zero coefficient in the nonbonding MO. For a normalized MO, $y=1 / \sqrt{12}$.

structures in this list (with the exception of altanpyracylene)-as with altan-corannulene (1) and altancoronene (2)-were found to have a single, unoccupied, nonbonding orbital, with nonzero coefficients on alternating centers (and also alternating in sign) only around the perimeters of these mono-altan systems. In addition it should be noted that none of the parent structures listed (with the exception of pyracylene, ${ }^{6}$ which has one nonbonding MO) has any nonbonding MOs. That is to say, all the parent structures (with the exception of pyracylene) have a nullity ${ }^{46}$ of zero, while the mono-altans of 9 of the 10 structures listed (all of them (7-16), but excluding the altan of pyracylene (16)) have a nullity of 1 . Studies on the mathematical properties of the altans in general have shown that the mono-altans of parent structures that have a nullity of zero themselves have a nullity of $1,^{8-10,46}$ and this is borne out in practice in the case of the calculations that we have carried out on $\mathbf{1}$ and $\mathbf{2}$ and the altans of the nine other conjugated systems (7-15) that were examined (and illustrated) in refs 6, 7, and 22 and which are listed above.

We also observe and note here that, as was the case with $1-$ 6, in each of these nine mono-altans (7-15) discussed in refs 6,7 , and 22, this single nonbonding MO is likewise the LUMO in the neutral species and the HOMO in the corresponding dianion. Furthermore, as was the case with these nine monoaltans (7-15), the following were found:

(i) In the case of each neutral altan (7-15) and its corresponding dianion, although ring currents were very different between the two, the bond currents in all internal bonds were precisely the same in the dianion as in the neutral species, but the bond currents flowing around the perimeter were found to be different in the neutral species from the corresponding bond currents in the dianion.

(ii) In all the listed cases, the lone nonbonding orbital in the neutral species was unoccupied as the LUMO, whereas that orbital in the corresponding dianion received and accommodated the two extra $\pi$-electrons (with paired spins) added in the course of applying the Aufbau process ${ }^{14,37}$ and so became the HOMO in the dianion, in an analogy with Figure 10. 
(iii) That nonbonding $\mathrm{MO}$, in every case, had nonzero coefficients centered only on certain (alternate) carbon atoms on the periphery-the coefficients centered on every other carbon atom on the periphery were zero, and the intermediate ones alternated as $x$ and $-x, y$ and $-y$, etc. as displayed, for example, in the diagrams in Figure 11 (for the nonbonding orbital in 1) and Figure12 (for the nonbonding orbital in 6).

(iv) In contrast to all of the other species mentioned here (7-15), there is a different situation when pyracylene (16) is the parent structure, as it already has a (single) nonbonding $\mathrm{MO}$, even before the altanization process begins; the altan of pyracylene thus has two nonbonding MOs. ${ }^{8-10,46}$ In the ground state of the neutral altan of pyracylene, the two nonbonding orbitals are left unoccupied, after the Aufbau process has been applied. ${ }^{14,37}$ These two nonbonding orbitals are in fact, therefore, both LUMOs in the neutral species, as with $1-15$. When the transition from the neutral species to the dianion is effected, however, one of these two extra electrons that are added during the course of the Aufbau process when the dianion is formed from the neutral species should go into each of the two degenerate nonbonding levels. This would then lead to a triplet ground state, and in that situation, there would be infinite paramagnetism ${ }^{39,47}$ and so the ring-current calculation, although viable for the neutral mono-altan 16indeed, we reported it (labeled as "structure 5") in ref 6could not be carried out for the dianion of that mono-altan, $16^{2-}$.

Effect on the Coulson Bond Orders $\left(P_{r s}\right)$ and the Imaginary Bond-Bond Polarizabilities $\left(\overline{\boldsymbol{\pi}}_{(r s)(t u)}\right)$ of Going from the Neutral Species to the Dianion in Structures 1-15. It is clear from the definition of $P_{r s}$ in eq 1 that, in the dianions, the contributions from the two extra $\pi$-electrons in the nonbonding orbital to the bond orders of any bond between two adjacent atoms on the periphery will identically be zero; this is because precisely one of the LCAO-MO coefficients centered on carbon atoms $r$ and $s$ that form a bond on the periphery will necessarily be zero, because of the rule that, in a nonbonding orbital, the LCAO coefficients centered on all carbons atoms that are adjacent to a given carbon atom must necessarily sum to zero-please see, for example, the bottom of page 100 of ref 14 and see also Figures 11 and 12 for examples of this. It is also straightforward to see from eq 1 that, in the dianions, the contributions from the two extra $\pi$ electrons in the nonbonding orbital to the bond orders of (a) any bond between two adjacent interior carbon atoms and (b) any bond between a carbon atom on the periphery and an adjacent internal carbon atom will also identically be zero.

The imaginary bond-bond polarizabilities, $\bar{\pi}_{(r s)(t u)}$, on the other hand, are calculated from eqs 2 and 3. From these it is evident from a consideration of, for example, Figures 11 and 12 , that in the dianion the contributions from the extra two electrons in the nonbonding orbital to the mutual bond-bond polarizabilities between two bonds, both of which are internal, will still always be identically zero. But whether there are necessarily zero or nonzero contributions from the two extra $\pi$ electrons in the nonbonding level to the mutual bond-bond polarizabilities (a) between two bonds on the periphery or (b) between a peripheral bond and an entirely internal one is not obvious in the general case from eqs 3 and 4 . This is the crux of the matter at issue.

\section{CONCLUDING REMARKS}

In principle, therefore, the magnetic behavior of the monoaltans considered here ( 1 and $\mathbf{2}$ in Figures 4 and 5) and 7-15 (studied and illustrated in refs 6, 7, and 22) can be rationalized intuitively: because the nonbonding orbitals have nonzero LCAO-MO coefficients only for certain atoms around the periphery, only the bond currents in bonds on that perimeter will have the possibility of varying, as the transition is made from the neutral altan to the dianion of it. This is why the calculated ring currents in the neutral species and the corresponding dianion are different in these structures (see, for example, Figures 4 and 5).

In the case of the iterated altans-the $(\text { altan })^{2}$-structures (3 and 4) and the (altan $)^{3}$-structures (5 and 6)-our calculations have shown that all bond currents (even those in bonds on the periphery) are exactly the same in the dianion as in the corresponding neutral species. This is because, unlike in the cases of $\mathbf{1}$ and $\mathbf{2}$ and the altans of 7-15 discussed in refs 6, 7, and 22, actual calculation on 3-6 indicates no change in any of the imaginary bond-bond polarizabilities (eqs 2 and 3 ) when going from the neutral species to the corresponding dianion. In addition, as argued, there is of course no change in the Coulson bond orders (eq 1) when that transition from neutral species to dianion is made. Accordingly, the ring currents, and by extension the bond currents, are identical in the neutral species and the corresponding dianion for the iterated altans 3-6.

Future developments of these studies that might possibly be fruitful could include the following:

(i) To extend the HLPM investigations to encompass even higher iterated altans, such as (altan $)^{n}$-corannulene and (altan) $)^{n}$-coronene (with $n>3$ ). This might, however, lead to the sort of practical difficulties concerning round-off error that were raised in section Numerical Accuracy of Reported Calculations.

(ii) To encourage practitioners of ab initio approaches (such as those reported in refs 1-4) to investigate structures 3-6 and their dianions. More sophisticated ipso-centric ab initio calculations might also possibly confirm the remarkable and unexpected predictions that have arisen here in the context of the pseudo graph-theoretical HLPM formalism ${ }^{18,19,21-24}$ concerning the identical ring-current and bond-current maps in the neutral iterated altans 3-6 and their respective dianions that have been examined here.

There are already indications that point (ii) may yield conclusions of interest by virtue of some of the current strengths calculated by use of the ab initio ipso-centric method for a limited number of internal bonds in $\mathbf{1}$ and $\mathbf{1}^{2-}$, which have been reported by Monaco and Zanasi in ref 4 . For the internal bonds labeled a, b, c, and d in Scheme 3 on page 735 of ref 4 , the values of integrated current strengths (the equivalent of our bond currents) are stated to be as follows (for bonds a, b, c, and d, respectively, with neutral species 1 first, followed by $1^{2-}$ in parentheses): $-0.45(-0.47), 0.00$ $(0.00), 0.81(0.85)$, and $0.76(0.82)$. It should immediately be conceded, however, that the pair of zero currents (for bond $b$ ) are guaranteed to agree by symmetry, irrespective of the approximations and assumptions made in the calculation. Nevertheless, given that bond currents arising from $a b$ initio calculations (unlike our HLPM bond currents) do not in general rigorously obey Kirchhoff's Conservation $\mathrm{Law}^{38}$ at junctions (except in the idealized limit of a complete basis 
set) ${ }^{48}$ the approximate equality between the above corresponding pairs of internal bonds in $\mathbf{1}$ and $\mathbf{1}^{2-}$ is sufficiently encouraging to suggest that investigating the magnetic properties of the iterated altans 3-6 and their dianions by the ipso-centric ab intitio methods might prove to be worthwhile.

\section{AUTHOR INFORMATION}

\section{Corresponding Author}

*E-mail: tkd25@cam.ac.uk. Phone: +44 1223763811.

ORCID

Timothy K. Dickens: 0000-0003-0342-3597

Notes

The authors declare no competing financial interest.

\section{ACKNOWLEDGMENTS}

We are grateful to Mr. C. W. Haigh for helpful discussions and correspondence on symmetry and nonbonding orbitals. R.B.M. thanks the Master and Fellows of Peterhouse, Cambridge, for his election as a Visiting Fellow for Easter Term 2018, in order to collaborate with T.K.D. on this Article and on other work related to it.

\section{REFERENCES}

(1) Monaco, G.; Zanasi, R. On the Additivity of Current Density in Polycyclic Aromatic Hydrocarbons. J. Chem. Phys. 2009, 131, 044126.

(2) Monaco, G.; Zanasi, R. Three Contra-Rotating Currents from a Rational Design of Polycyclic Aromatic Hydrocarbons: AltanCorannulene and Altan-Coronene. J. Phys. Chem. A 2012, 116, 9020-9026.

(3) Monaco, G.; Memoli, M.; Zanasi, R. Additivity of Current Density Patterns in Altan-Molecules. J. Phys. Org. Chem. 2013, 26, 109-114.

(4) Monaco, G.; Zanasi, R. Anionic Derivatives of AltanCorannulene. J. Phys. Org. Chem. 2013, 26, 730-736.

(5) Dickens, T. K.; Mallion, R. B. Topological Hückel-LondonPople-McWeeny Ring-Currents and Bond-Currents in AltanCorannulene and Altan-Coronene. J. Phys. Chem. A 2014, 118, 933-939.

(6) Dickens, T. K.; Mallion, R. B. $\pi$-Electron Ring-Currents and Bond-Currents in some Conjugated Altan-Structures. J. Phys. Chem. A 2014, 118, 3688-3697.

(7) Dickens, T. K.; Mallion, R. B. Topological Ring-Current and Bond-Current Properties of the Altans of Certain K-Factorizable Conjugated Systems Containing "Fixed" Single-Bonds. J. Phys. Chem. A 2015, 119, 5019-5025.

(8) Gutman, I. Topological Properties of Altan-Benzenoid Hydrocarbons. J. Serb. Chem. Soc. 2014, 79, 1515-1521.

(9) Gutman, I. Altan Derivatives of a Graph. Iran. J. Math. Chem. 2014, 5, 85-90.

(10) Bašić, N.; Pisanski, T. Iterated Altans and their Properties. MATCH Commun. Math. Comput. Chem. 2015, 24, 653-666.

(11) Zanasi, R.; Della Porta, P.; Monaco, G. The Intriguing Class of Altan-Molecules. J. Phys. Org. Chem. 2016, 29, 793-798.

(12) Liu, C.; Sandoval-Salinas, M. E.; Hong, Y.; Gopalakrishna, T. Y.; Phan, H.; Aratani, N.; Herng, T. S.; Ding, J.; Yamada, H.; Kim, D.; et al. Macrocyclic Polyradicaloids with Unusual Super-Ring Structure and Global Aromaticity. Chem 2018, 4, 1586-1595.

(13) Steiner, E.; Soncini, A.; Fowler, P. W. Full Spectral Decomposition of Ring Currents. J. Phys. Chem. A 2006, 110, 12882-12886.

(14) Coulson, C. A.; O’Leary, B.; Mallion, R. B. Hückel Theory for Organic Chemists; Academic Press: London, 1978.

(15) London, F. Théorie Quantique des Courants Interatomiques dans les Combinaisons Aromatiques. J. Phys. Radium 1937, 8, 397409.
(16) Pople, J. A. Molecular Orbital Theory of Aromatic Ring Currents. Mol. Phys. 1958, 1, 175-180.

(17) McWeeny, R. Ring Currents and Proton Magnetic Resonance in Aromatic Molecules. Mol. Phys. 1958, 1, 311-321.

(18) Mallion, R. B. Topological Ring-Currents in Condensed Benzenoid Hydrocarbons. Croat. Chem. Acta 2008, 81, 227-246.

(19) Balaban, A. T.; Dickens, T. K.; Gutman, I.; Mallion, R. B. Ring Currents and the PCP Rule. Croat. Chem. Acta 2010, 83, 209-215.

(20) Trinajstić, N. Chemical Graph Theory, 2nd ed.; CRC Press: Boca Raton, FL, 1992; Chapters 4 and 6; pp 41-59 and 85-123.

(21) Dickens, T. K.; Mallion, R. B. Topological Ring-Currents in Conjugated Systems. MATCH Commun. Math. Comput. Chem. 2016, 76, 297-356.

(22) Dickens, T. K.; Mallion, R. B. An Analysis of Topological RingCurrents and their Use in Assessing the Annulene-Within-anAnnulene Model for Super-Ring Conjugated Systems. Croat. Chem. Acta 2013, 86, 387-406.

(23) Gomes, J. A. N. F.; Mallion, R. B. Aromaticity and Ring Currents. Chem. Rev. 2001, 101, 1349-1383 (especially pp 13581366).

(24) Gomes, J. A. N. F.; Mallion, R. B. The Concept of Ring Currents. In Concepts in Chemistry; Rouvray, D. H., Ed.; Research Studies Press Limited: Taunton, Somerset, England, United Kingdom, 1997 and John Wiley \& Sons, Inc.: New York, 1997; Chapter 7, pp 205-253.

(25) Monaco, G. On the Diatropic Perimeter of Iterated AltanMolecules. Phys. Chem. Chem. Phys. 2015, 17, 28544-28547.

(26) Haigh, C. W.; Mallion, R. B.; Armour, E. A. G. Proton Magnetic Resonance of Planar Condensed Benzenoid Hydrocarbons. II A Critical Evaluation of the McWeeny "Ring Current" Theory. Mol. Phys. 1970, 18, 751-766.

(27) Mallion, R. B. $\pi$-Electron "Ring Currents" in Fluoranthene and Related Molecules. J. Mol. Spectrosc. 1970, 35, 491-493.

(28) Gershoni-Poranne, R.; Gibson, C. M.; Fowler, P. W.; Stanger, A. Concurrence Between Current Density, Nucleus-Independent Chemical Shifts, and Aromatic Stabilization Energy: the Case of Isomeric [4]- and [5] Phenylenes. J. Org. Chem. 2013, 78, 7544-7553.

(29) Oziminski, W. P.; Palusiak, M.; Dominikowska, J.; Krygowski, T. M.; Havenith, R. W.; Gibson, C. M.; Fowler, P. W. Capturing the Elusive Aromaticity of Bicalicene. Phys. Chem. Chem. Phys. 2013, 15, 3286-3293.

(30) Fowler, P. W.; Cotton, S.; Jenkinson, D.; Myrvold, W.; Bird, W. H. Equiaromatic Benzenoids: Arbitrarily Large Sets of Isomers with Equal Ring-Currents. Chem. Phys. Lett. 2014, 597, 30-35.

(31) Ceulemans, A. J. Group Theory Applied to Chemistry; Theoretical Chemistry and Computational Modelling; Springer-Verlag: Dordrecht, Heidelberg, New York, and London, 2013.

(32) When the conjugated systems dealt with in this study are viewed as three-dimensional planar structures, the symbols $D_{5 h}$ and $D_{6 h}$ are appropriate. These are groups of order 20 and 24, respectively. ${ }^{31}$ Because our ring-current calculations are using Hückel theory, ${ }^{14}$ which involves $\pi$-orbitals, the molecular planes are significant and our calculations are thus being carried out within these groups. However, if these species were being considered purely as graphs, ${ }^{20}$ the situation would be different. The molecular plane is, in this context, now irrelevant. In those structures with 5-fold symmetry, the five 2 -fold rotation axes and the five mirror planes have exactly the same effect on the graph vertices. So, the relevant group would be $C_{5 v}$ (of order 10) and-for those previously classified as $D_{6 h}-C_{6 v}$ (of order 12). We are very grateful to Mr. C. W. Haigh for kindly clarifying this distinction.

(33) Haigh, C. W.; Mallion, R. B. Proton Magnetic Resonance of Non-Planar Condensed Benzenoid Hydrocarbons. II. Theory of Chemical Shifts. Mol. Phys. 1971, 22, 955-970.

(34) Antić, M.; Furtula, B.; Radenković, S. Aromaticity of Nonplanar Fully Benzenoid Hydrocarbons. J. Phys. Chem. A 2017, 121, 36163626.

(35) Gayoso, J.; Boucekkine, A. Sur le Calcul de la Susceptibilité Diamagnétique des Systèmes $\pi$, dans le Cadre de Hückel, au Moyen 
de la Technique des Perturbations. C. R. Acad. Sci. (Paris) C 1971, 274, 184-187.

(36) Mallion, R. B. Some Graph-Theoretical Aspects of Simple Ring Current Calculations on Conjugated Systems. Proc. R. Soc. London, Ser. A 1975, 341, 429-449.

(37) Mallion, R. B.; Rouvray, D. H. Molecular Topology and the Aufbau Principle. Mol. Phys. 1978, 36, 125-128.

(38) Kirby, E. C.; Mallion, R. B.; Pollak, P.; Skrzyński, P. J. What Kirchhoff Actually Did Concerning Spanning Trees in Electrical Networks and its Relationship to Modern Graph-Theoretical Work. Croat. Chem. Acta 2016, 89, 403-417.

(39) Pople, J. A.; Untch, K. G. Induced Paramagnetic Ring Currents. J. Am. Chem. Soc. 1966, 88, 4811-4815.

(40) Steiner, E.; Fowler, P. W. Four- and Two-electron Rules for Diatropic and Paratropic Ring Currents in Monocyclic $\pi$-Systems. Chem. Commun. 2001, 2220-2221.

(41) Jung, D. E. Grenzen der Ringstromdefinition der Aromatizität: Ringstromberechnungen in nicht-alternierenden Tri- und Tetracyclen. Tetrahedron 1969, 25, 129-134.

(42) Mallion, R. B. Some Comments on the Use of the "RingCurrent" Concept in Diagnosing and Defining "Aromaticity". Pure Appl. Chem. 1980, 52, 1541-1548.

(43) Mallion, R. B. Ring Current Effects in $C_{60}$. Nature 1987, 325, $760-761$.

(44) Dickens, T. K.; Mallion, R. B. Ring-Current Properties of Bispentalenes and Related Structures - Comparison of $A b$ Initio and Hückel-London-Pople-McWeeny (HLPM) “Topological” Calculations. Croat. Chem. Acta 2017, 90, 369-382.

(45) Balaban, A. T.; Bean, D. E.; Fowler, P. W. Patterns of Ring Currents in Coronene Isomers. Acta Chim. Slov. 2010, 57, 507-512.

(46) Gutman, I.; Borovićanin, B. Nullity of Graphs: An Updated Survey. In Applications of Graph Spectra; Cvetković, D., Gutman, G., Eds.; Mathematical Instititute Belgrade: Belgrade, 2011; pp 137-154.

(47) Aihara, J. Magnetic Resonance Energy and Topological Resonance Energy. Phys. Chem. Chem. Phys. 2016, 18, 11847-11857.

(48) Epstein, S. T. Gauge Invariance, Current Conservation and GIAOs. J. Chem. Phys. 1973, 58, 1592-1595. 